\title{
Control estructural de las mineralizaciones de barita y cobre en los corregimientos de Caracolí y Camperucho, Cesar, Colombia
}

\author{
Amat D. Zuluaga ${ }^{1 *}$; Wilfar Torrenegra ${ }^{1}$; Frank Lascarro ${ }^{1}$
}

DOI: http://dx.doi.org/10.18273/revbol.v41n1-2019004@ @ @

Forma de citar: Zuluaga, A.D., Torrenegra, W., y Lascarro, F. (2019). Control estructural de las mineralizaciones de barita y cobre en los corregimientos de Caracolí y Camperucho, Cesar, Colombia. Boletín de Geología, 41(1), 85-96. DOI: 10.18273/revbol.v41n1-2019004.

\section{RESUMEN}

Entender los controles estructurales es fundamental para la comprensión de los emplazamientos de fluidos mineralizantes, pues las estructuras geológicas desarrollan un papel como canales en el transporte y precipitación de minerales. El área de estudio comprende la zona rural del corregimiento de Caracolí, donde afloran mineralizaciones de Barita y la zona rural del corregimiento de Camperucho, donde afloran mineralizaciones de Cobre, ambas ubicadas al sur del municipio de Valledupar, Cesar, Colombia. La recopilación de datos estructurales en campo y su análisis evidencian un control tectónico definido de las mineralizaciones a lo largo de un sistema de fallas orientadas NEE-SWW determinado como N71E/81SE que cortan secuencias volcánicas y volcanosedimentarias asociadas a la Formación Guatapurí, la Unidad Riodacita de los Tábanos y Espilitas Triásicas. Estas fracturas permitieron el ascenso de fluidos hidrotermales enriquecidos en bario (Caracolí) generando vetas de barita y la filtración de aguas meteóricas formando un enriquecimiento secundario de cobre (Camperucho) representados en óxidos y carbonatos de cobre. El control estructural del área es definido por fallas inversas con componente de rumbo generando movimientos sinestrales, producto de esfuerzos de cizalla simple con las siguientes componentes: $\sigma 1 \mathrm{y}$ $\sigma 3$ en direcciones NNE y SEE respectivamente. Las mineralizaciones de barita se dan a partir de esfuerzos compresivos y de cizalla medidos en campo generando fallas inversas con componente de rumbo sinestral, permitiendo el emplazamiento de fluidos mineralizantes en formas de "rosario" y "bolsones" observadas a lo largo de estas zonas de debilidad. Por otra parte, las mineralizaciones de óxidos de Cobre son generados por enriquecimientos secundarios de litologías volcanosedimentarias con grado bajo en contenido de cobre a través de zonas de fallas que permiten la filtración de aguas meteóricas.

Palabras clave: Control estructural; diaclasa; falla; mineralización.

\section{Structural control of barite and copper mineralization at the district of Caracolí and Camperucho, Cesar, Colombia}

\begin{abstract}
Understanding structural controls is essential for understanding the sites of mineralized fluids, since geological structures develop a role as channels in the transport and precipitation of minerals. The study area comprises the rural area of the Caracolí district where outcrop mineralization of Barite and the rural area of the Camperucho where Copper mineralizations outcrops, both located to the south of the municipality of Valledupar, Cesar, Colombia. The analysis of the data collected on field showed a definite tectonic control of the mineralizations along a system of oriented faults NEE-SWW determined as N71E/81SE cutting volcanic and volcanosedimentary sequences associated with the Guatapurí formation, the Rhyodacite of the Tabanos and Espilitas Triassic units. These fractures allowed the rise of hydrothermal fluids enriched in barium (Caracolí) forming veins of barite, and the filtering of meteoric waters generating a secondary copper mineralization (Camperucho) represented on copper oxide and carbonate minerals. The structural control of the area is defined by inverse faults with sinistral movement product of simple shearing efforts with the following directions: $\sigma 1$ and $\sigma 3$, NNE and SEE respectively. The mineralizations of barite are emplaced from compressive and shear efforts, measured on field generating inverse faults with sinistral movement allowing the siting of mineralizing fluids with "rosario" and "bolsons" shape, observed in the field along these areas of weakness. On the other hand, the copper oxide deposits are generated by secondary enrichment of volcanosedimentary litholigies with low grade of copper content throughout fault planes which allowed the filtration of meteorics water.
\end{abstract}

Keywords: Structural control; joints; fault; mineralization.

${ }^{1}$ Grupo de Investigación en Ingeniería Geológica (GIINGEO), Escuela de Ingeniería Geológica, Fundación Universitaria del Área Andina, Valledupar, Colombia. (*) azuluaga16@areandina.edu.co; witorrenegra@areandina.edu.co; flascarro@areandina.edu.co 


\section{INTRODUCCIÓN}

Los corregimientos de Caracolí y Camperucho (FIGURA 1) son zonas en las que afloran vetas de minerales de interés económico como la barita y el cobre, asociadas a eventos de circulación de fluidos y alojadas en rocas volcánicas y volcanosedimentarias (Tschanz et al., 1969) de edad Triásico-Jurásicas asociadas a la Formación Guatapurí, la Unidad Riodacita de los Tábanos y Espilitas Triásicas. Según Ortega (2011), en este período existieron episodios volcánicos que restringieron las mineralizaciones a eventos posteriores a la litificación de las rocas involucradas. Las mineralizaciones de Barita se emplazaron dentro y en dirección de algunas estructuras regionales producto de esfuerzos compresivos y de cizalla (fallas y fracturas asociadas), siendo los emplazamientos controlados por fallas premineralización, generando espacios qué, aunque apretados, permitieron el relleno una vez efectuada la inyección y precipitación de los fluidos mineralizantes asociados a Barita. Por otra parte, las mineralizaciones de cobre tienen un origen hidrotermal inicialmente evidenciado en mineralizaciones hipógenas de bornita y calcopirita, posteriormente sufren un enriquecimiento secundario supergénico alterno que favorecieron el enriquecimiento secundario de cobre, donde las fallas jugaron el rol de controlar estructuralmente las mineralizaciones, convirtiéndose en vías de fácil filtración de aguas meteóricas, para generar el enriquecimiento secundario de óxidos y carbonatos de cobre a partir de rocas volcanosedimentarias que ya contenían una concentración baja de éste (Corredor y Mendoza, 2016). Ortega et al. (2014), define mineralizaciones de cobre en la Serranía del Perijá (al oriente de la zona de estudio) con composición mineral, origen y patrones estructurales muy similar a la estudiada en este trabajo, lo cual nos permite correlacionar los resultados.

Hasta el momento, las mineralizaciones de barita se vinculan a un evento plutónico Cenozoico (Maestre et al., 2014), no obstante, la ausencia de evidencias petroquímicas y de edades no permiten relacionar de forma directa las mineralizaciones con cuerpos asociados a algún evento de este tipo. Este trabajo es el primero en abordar y establecer descriptivamente una relación entre las mineralizaciones de barita y cobre y los dominios estructurales regionales y locales.

En este trabajo se estudiaron las mineralizaciones y su relación con las estructuras locales y regionales, efectuándose un análisis estructural basado en el compendio de datos estructurales, su respectivo análisis, y descripción de las características morfológicas de los emplazamientos, con la finalidad de considerar aspectos como, posible distribución y significado geológico de las mineralizaciones; información relevante al momento de efectuar análisis regionales y llevar a cabo el desarrollo de la prospección y exploración de estas menas minerales; contribuyendo además al conocimiento geológico y mineralógico del depósito mismo.

\section{METODOLOGÍA}

La investigación se efectúa mediante el desarrollo de diversas etapas en las que se realizó: un análisis bibliográfico detallado, haciendo distinción en la geología estructural regional y local de la zona de estudio, en las unidades litológicas y geomorfológicas y en investigaciones análogas a nuestra área. Posteriormente se planeó y ejecutó la campaña en campo teniendo como base los mapas geológicos de la plancha 33 (Colmenares et al., 2007) y los planos topográficos 33-IV-A (IGAC, 2005a) y 33-IV-C a escala 1:25000 (IGAC, 2005b), donde se hizo el levantamiento geológico de 856 datos estructurales utilizando brújulas marca Brunton, distribuidos así: 759 diaclasas, 10 planos de falla, 61 venas, 12 diques, 4 datos cinemáticos de fallas y 10 de estratificación. El levantamiento se realizó a escala 1:500 en las zonas de interés (a lo largo de planos de falla, frentes de explotación y afloramientos de estructuras geológicas). Posteriormente, los datos fueron agrupados y clasificados en el software Microsoft Excel de donde fueron exportados a los softwares Rocscience, Dip versión 6.008, y FaultKin - FaultKinWin versión 7.5.0 codificados y creados por Allmendinger et al. (2012) y Marrett y Allmendinger (1990), de manera separada. A partir las proyecciones estereográficas arrojadas en el análisis de datos del software, se inició la interpretación de los datos, analizando primeramente de manera independiente cada tipo de dato tomado y de cada zona estudiada, es decir, las venas en la zona de Caracolí fueron analizadas independiente de las venas de la zona de Camperucho y de esa manera para los demás tipos. Una vez hecho el análisis por separado, se compararon los resultados de cada tipo entre zonas, por ejemplo, resultados de venas de Camperucho con venas de Caracolí. Finalmente, se analizó la relación de un tipo de dato con otro en ambas zonas, es decir, la relación entre venas y diaclasas de Caracolí, fallas y diques de Camperucho, diques de Caracolí con fallas de Camperucho, etc. Como resultado, se definieron las orientaciones principales y secundarias, la cinemática de las fallas y las direcciones de los esfuerzos que actuaron para generar los trenes estructurales actuales. 
Por último, a partir de la interpretación realizada, se relacionan las estructuras encontradas, orientaciones y geometrías con las mineralizaciones de barita y cobre, con el fin de contribuir al conocimiento geológico del área y realizar exploraciones más eficaces para los titulares mineros de la región.

\section{GEOLOGÍA REGIONAL}

En un contexto regional el área de estudio se encuentra ubicada en el Terreno Sierra Nevada de Santa Marta, con un estilo estructural de Fallamiento de Bloques, estructuralmente limitado al norte por la Falla de Oca, al oeste por la Falla de Santa Marta y al este por la Falla del Cesar, está compuesta por tres cinturones tectonoestratigráficos alineados con dirección NE-SW (Bayona et al., 2007). Montes et al. (2010), sugiere que existió una rotación de bloques que permitió movimientos dextro laterales de la falla de Oca y sinestro laterales de la falla Santa Marta-Bucaramanga. Litológicamente está constituido por rocas Volcánicas que comprenden edades Triásico-Jurásicas (Etayo et al., 1983).

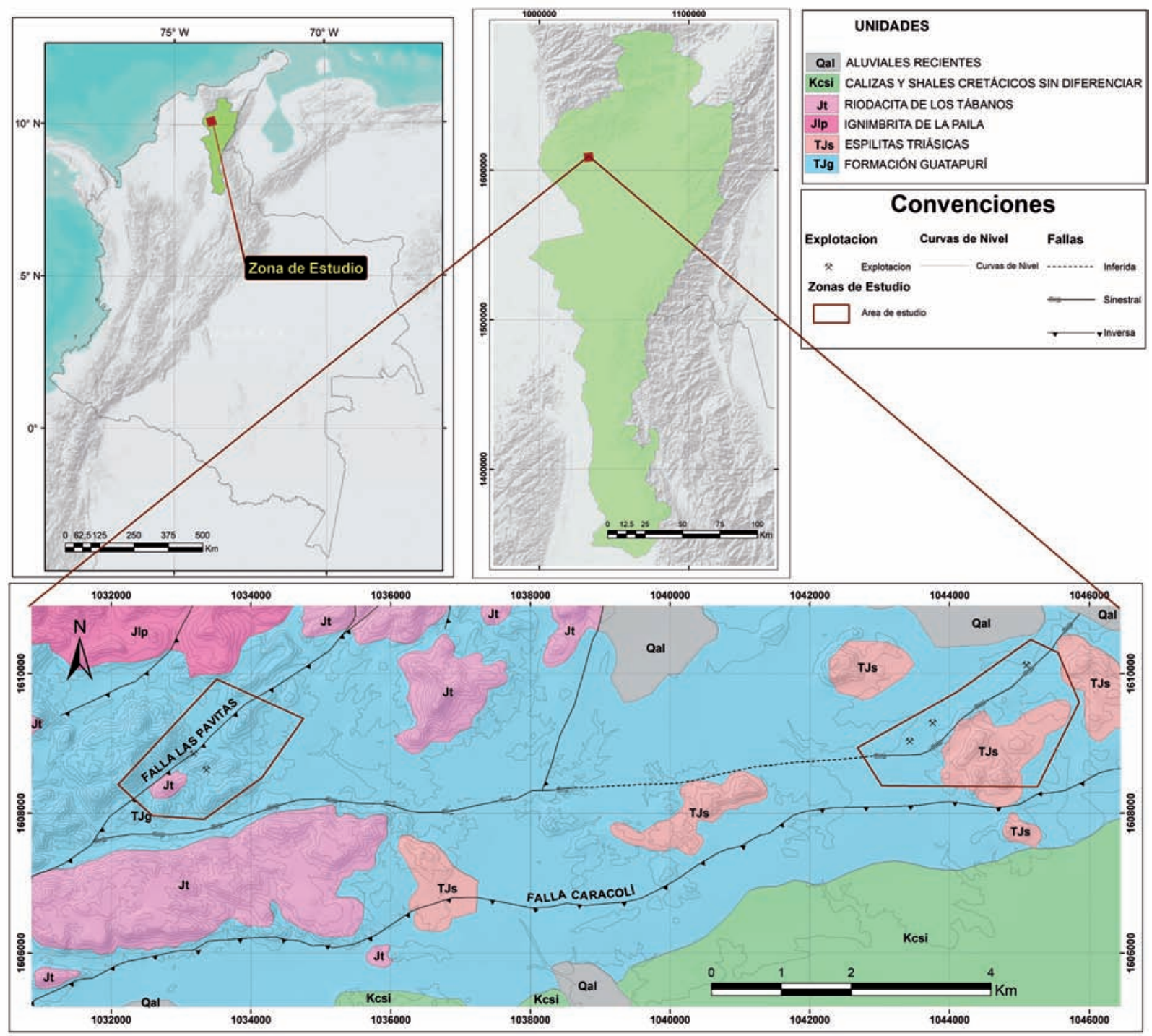

FIGURA 1. Mapa de localización de área de estudio. El recuadro izquierdo corresponde a la zona de Camperucho y el recuadro derecho la zona de Caracolí. La imagen muestra la localización de las diferentes unidades geológicas aflorantes en la zona, modificado de la plancha 33, escala 1:100 000 (Colmenares et al., 2007). 
Las mineralizaciones de barita y cobre según Corredor y Mendoza (2016), son un conjunto de mineralizaciones a partir de fluidos hidrotermales y que para el caso del cobre presenta también en algunas zonas con enriquecimiento secundario supergénico. Todas hospedadas en rocas volcanoclásticas y volcanosedimentarias Triásico-Jurásicas, cuya composición varía entre lodolitas e ignimbritas, que cubren casi en su totalidad el área de estudio. Las lodolitas corresponden a la Fm. Guatapurí (TJg) (Tschanz et al., 1969; Tschanz et al., 1974), constituidas por un conjunto de rocas acumuladas por procesos de disgregación de litologías formadas por procesos eruptivos lávicos y flujos piroclásticos de color rojizo, que se depositaron al oriente y sur de la Sierra Nevada de Santa Marta, por otra parte, las ignimbritas corresponden a la Fm. Riodacita de los Tábanos (Jt) y Espilita Triásicas (TJs), acumuladas allí por procesos eruptivos piroclásticos (Colmenares et al., 2007).

La Formación Guatapurí agrupa una serie de depósitos de color rojo, morado y purpura constituyéndose en superposiciones de secuencias granodecrecientes con interposiciones de rocas volcánicas y volcanoclásticas en contactos erosivos. Las Espilitas Triásicas refieren a un conjunto de rocas de apariencia basáltica de textura porfiroafanítica, cristales en un $10 \%$ euhedrales y en una matriz vítrea de color verdoso, cristales finos, de anfíboles y plagioclasa. Las Riodacitas de los Tábanos corresponden a rocas volcánicas de composición félsica y color rosado, con textura porfírica aflorantes como tobas latitícas, andesiticas, riodaciticas y líticas con fragmentos de rocas volcanoclásticas tamaño bloques (Colmenares et al., 2007).

En el corregimiento de Camperucho afloran mineralizaciones de óxidos de cobre rellenando fisuras, producto de enriquecimiento supergénico (secundario) al parecer relacionados con la percolación de fluidos meteóricos (Corredor y Mendoza, 2016). Los enriquecimientos supergénicos según Reich y Vasconcelos (2015), son acumulaciones secundarias in-situ, como resultado de tres procesos esenciales: oxidación electroquímica de sulfuros primarios, óxidos o minerales nativos; transporte del metal liberado como metal soluble; y precipitación de metales por reducción o por intercambio catiónico de la solución metálica.

\section{MARCO ESTRUCTURAL}

La Sierra Nevada de Santa Marta está subdividida en 6 regiones estructurales (FIGURA 2), con características geométricas y cinemáticas propias. A nivel regional se presentan estructuras geológicas producto de esfuerzos compresivos generados durante la deformación ocurrida por la exhumación de la Sierra Nevada de Santa Marta, que influyen de forma directa en el comportamiento de la mineralización a nivel local. La geología estructural del área ha sido muy poco analizada en detalle y con muy pocos trabajos de campo detallados, entre los estudios más relevantes son los realizados por Tschanz et al. (1969) y Colmenares et al. (2007), pero no alcanzan a detallar el comportamiento estructural local de la zona estudiada. En el área de trabajo se determinaron estilos estructurales dominados por la Falla las Pavitas (NW del área), caracterizadas por trazas suavemente sinuosa con rumbos entre N70E y N80E, y por la Falla Caracolí (SE del área) con una traza paralela a las Pavitas. Ambas estructuras afectando litologías TriásicoJurásicas (Colmenares et al., 2007). A nivel local son notorios algunos pequeños pliegues y cabalgamientos producto de dichos esfuerzos compresivos.

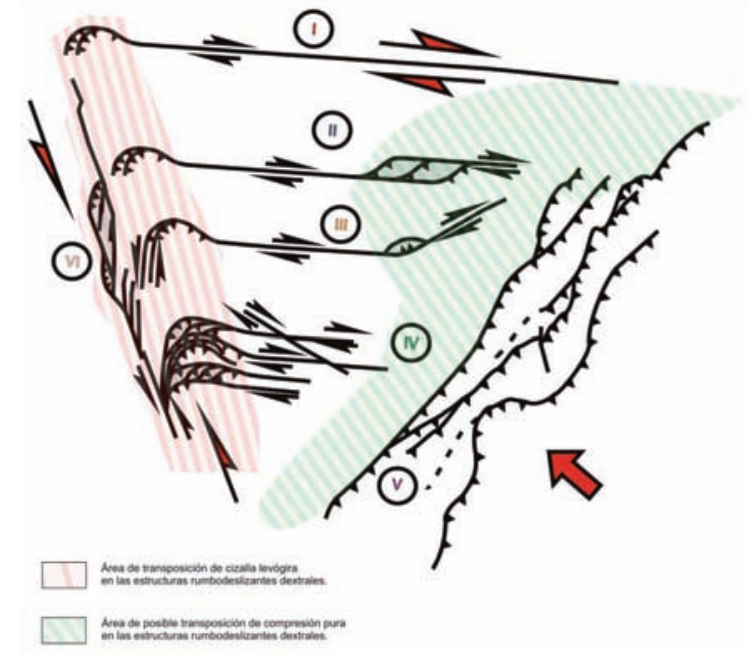

FIGURA 2. Transposición estructural de la Sierra Nevada de Santa Marta (no a escala). Tomado de Colmenares et al. (2007).

El área de trabajo está ubicada en la región IV en la cual según Colmenares et al. (2007) deduce que la geometría estructural de esta región es producto de la sobreimposición sincrónica de 2 estilos diferentes: transcurrencias dextro-laterales orientadas EW y transcurrencias levógiras orientadas NS o NNE, 
transposición que produce el arqueamiento estructural hacia el NW. Sin embargo, en el trabajo de campo solo se observaron estructuras levógiras orientadas NE-NEE y con un componente inverso con vergencia al SE.

\section{RESULTADOS}

El análisis estructural de las zonas mineralizadas se llevó a cabo examinando y evaluando el comportamiento de vetas, diaclasas y fallas reconocidas en campo. Los distintos planos estructurales registrados en campo fueron medidos en función de sus rumbos y buzamientos, dividiendo su análisis en diaclasas, fallas y estructuras mineralizadas o de relleno.

\section{Estructuras de relleno}

En el corregimiento de Caracolí afloran mineralizaciones de barita rellenando fisuras a lo largo de un sistema principal de fallamiento, donde se reconocen mineralizaciones de cuarzo "diente de perro" lo cual indica un origen hidrotermal, y pinchamientos continuos de barita a lo largo de los mismos planos de falla en forma de "rosario" y "bolsones"; Las mineralizaciones de óxidos de cobre en Camperucho son de tipo supergénico y están alojadas en un sistema de fallas inversas con orientación preferencial NESW y estructuras asociadas. En las zonas estudiadas se identificaron tres estructuras principales de relleno, las cuales son diques, vetas y fallas, que se describen a continuación.

Diques: corresponden a intrusiones de rocas de composición intermedia-máfica, con orientaciones preferenciales de N72E, buzamientos de 87SE y espesores que van desde $10 \mathrm{~s} 30 \mathrm{~cm}$ a $40 \mathrm{~cm}$, estos están localizados en el corregimiento de Caracolí.

Vetas: Los afloramientos de vetas minerales en el área de estudio de Camperucho corresponden a óxidos de cobre y carbonatos de cobre entre los cuales se tienen cuprita, azurita, malaquita. La existencia de vetillas en las zonas estudiadas corresponde a relleno de fracturas asociadas a sistemas de fallamiento principal con orientación preferencial NEE-SWW y NW-SE (FIGURA 3A).
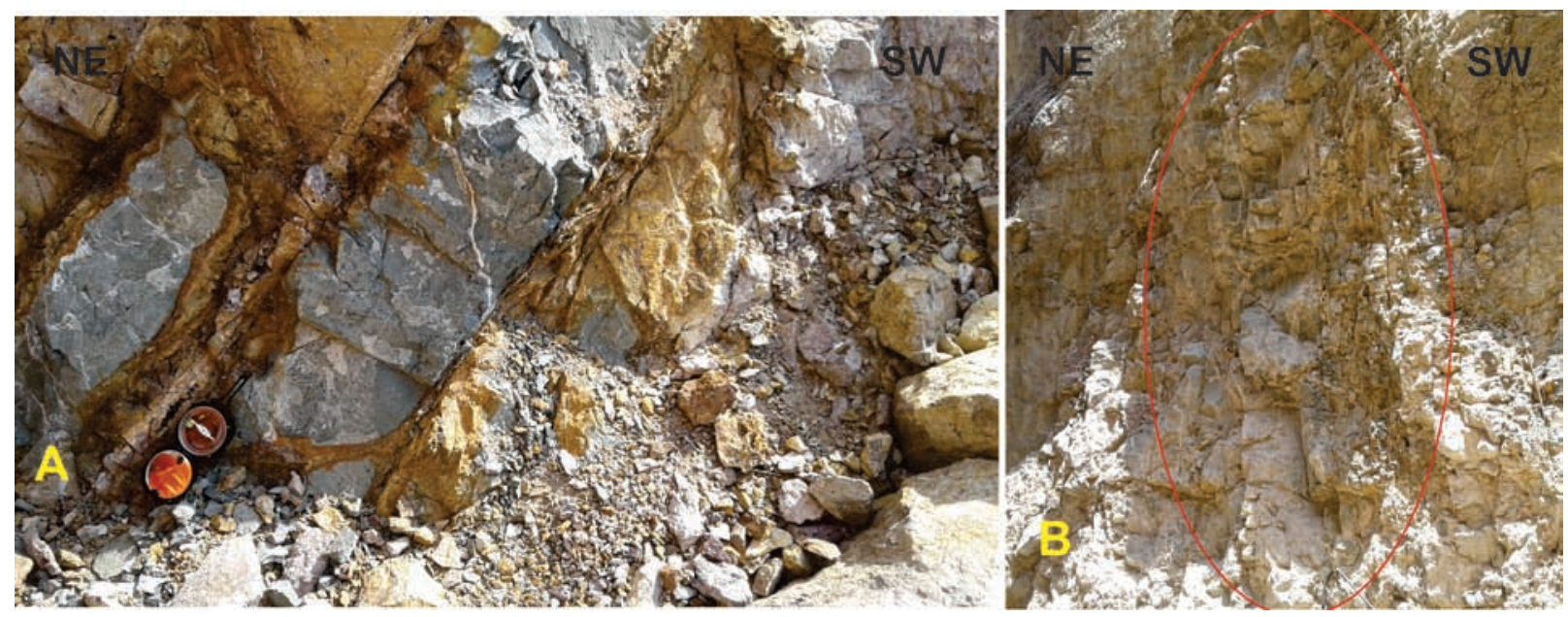

FIGURA 3. A. Afloramiento de rocas de la Unidad Riodacita de los Tábanos con presencia de estructuras hidrotermales asociadas al sistema de fallamiento principal perteneciente al área de estudio. B. Brechas de falla: bloques bastante fracturados en los que la barita envolvió los fragmentos generados por el movimiento de los bloques.

Se conoce que las mineralizaciones de barita son de naturaleza hidrotermal producto del ascenso de fluidos minerales que rellenaron fracturas existentes (Maestre et al., 2014); estos son apreciables a través de la correspondencia entre las paredes de las fracturas fácilmente observable a causa de la tensión generada por los fluidos (Ortiz, 1991).

En el corregimiento de Caracolí los fluidos ascendieron principalmente por planos de zonas de falla. Estas fallas además de evidencia de movimiento de bloques, lineamientos geomorfológicos y otros factores, generaron rocas de falla en la mayoría de los casos brechas. Estas se evidencian en las mineralizaciones de barita, las cuales cementaron los fragmentos generados por el movimiento de los bloques (FIGURA 3B). La mineralización se ve acompañada de minerales como calcita, fluorita y calcopirita en cantidades menores escasamente reconocibles.

En el corregimiento de Camperucho las mineralizaciones de óxidos y carbonatos de cobre son 
generados a partir de enriquecimiento secundario de la roca encajante. Esta presenta contenido previo de cobre diseminado, observado en minerales de naturaleza hipógena (calcopirita, bornita). El enriquecimiento secundario se da, por la removilización de cobre por aguas meteóricas a través de los planos de falla, las cuales presentan direcciones preferenciales NE-SW y vetillas asociadas a microfracturas tipo riedels en dirección NW-SE.

Fallas: Se reconocieron en ambos corregimientos fallas geológicas locales, las cuales se disponen como estructuras de relleno mineral principales. Los trazos de fallas observados se aprecian generalmente como planos de altos buzamientos $\left(>79^{\circ}\right)$ de dirección preferencial NEE-SWW, que cortan rocas volcanosedimentarias Triásico-Jurásicas de estructura masiva, presentan evidencias de indicadores cinemáticos tales como estructuras de cizalladura tipo riedels, estrías y escalerillas de falla. En el corregimiento de Caracolí, en los planos de fallas se aprecian rellenos de minerales de barita, fluorita y diques de rocas intermedias-máficas, observado en el frente de explotación de la cantera La Lucha (FIGURA 4). En Camperucho, como planos mineralizados por procesos supergénicos de malaquita y azurita, mostrando alteraciones a lo largo de los planos de falla. Las alteraciones son poco pervasivas (baja intensidad, no penetra significativamente la roca), no se observa más alteración después de $30 \mathrm{~cm}$ a lo ancho del plano de falla, sin evidencia observable en todos los puntos de un halo de alteración (FIGURA 5). Las orientaciones y buzamientos para el área de Caracolí y Camperucho son de N72E-78SE y N84E75SE respectivamente.

En el corregimiento de Caracolí, se midieron 637 diaclasas, determinando 5 familias diferentes: predominando con mayor frecuencia rumbos de diaclasas de N15E, N45E y N25W, presentándose en menor frecuencia N79E, N44W; los cuales presentan inclinaciones, que oscilan en buzamientos de $50^{\circ}, 20^{\circ}$, $71^{\circ}, 16^{\circ}$ y $71^{\circ}$ respectivamente.

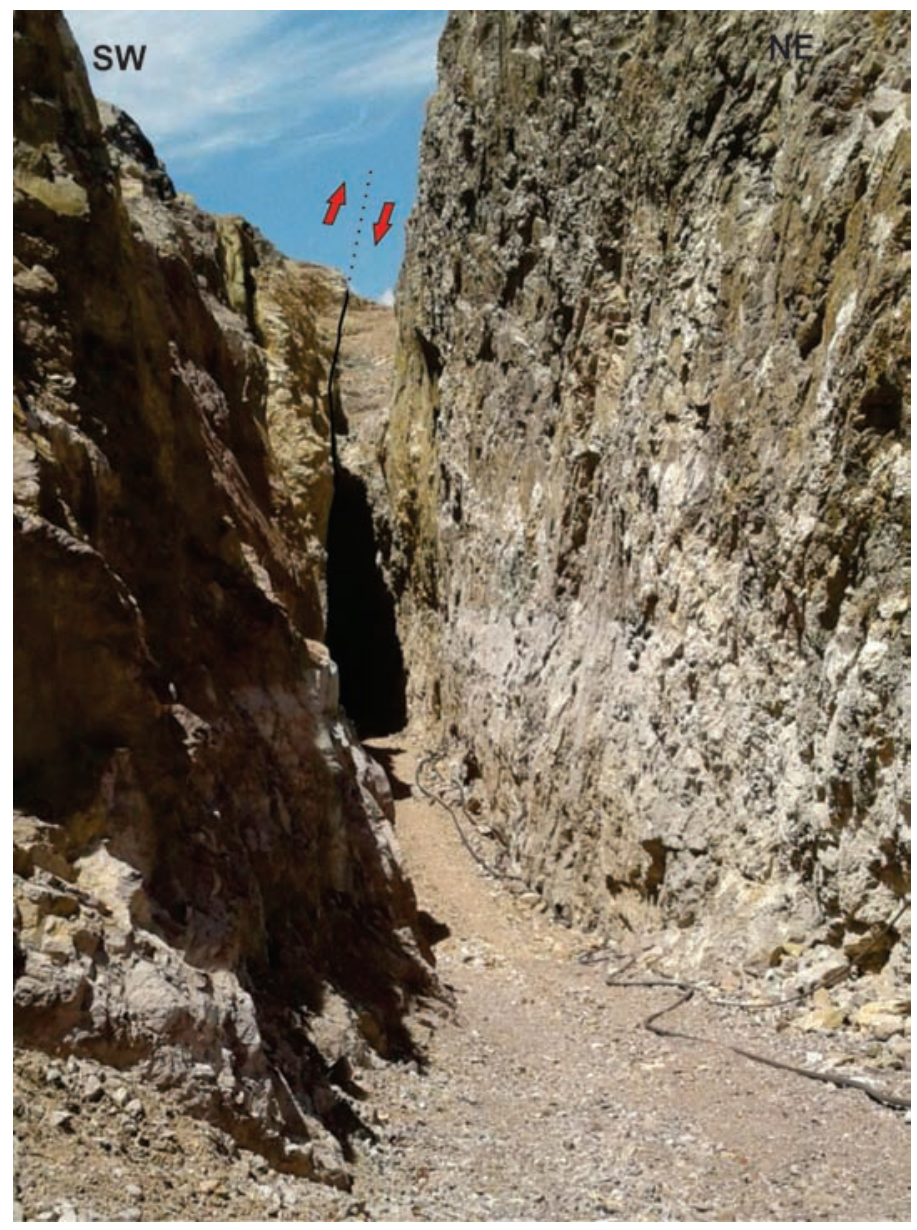

FIGURA 4. Aspecto del plano principal de la falla las Pavitas, localizado en el corregimiento de Caracolí en los frentes de explotación de la cantera La Lucha. 
En la zona de Caracolí, las fallas se evidencian en planos descubiertos en los distintos frentes de explotación junto con estructuras tipo riedels y estrías de falla, siendo en totalidad 2 planos de fallas principales observados y 3 secundarios asociados a los principales, con una orientación preferencial NEE-SWW: rumbos generales de $\mathrm{N} 69^{\circ}-74^{\circ} \mathrm{E}$ y altos buzamientos que oscilan en sus diferentes sectores en ángulos que van desde los $71^{\circ} \mathrm{y}$ $81^{\circ}$ y pequeñas fallas asociadas de menor buzamiento $\left(51^{\circ}-55^{\circ}\right)$ (FIGURA 6). El fallamiento y diaclasamiento en conjunto, corresponden a estructuras posteriormente rellenadas por fluidos ricos en bario.

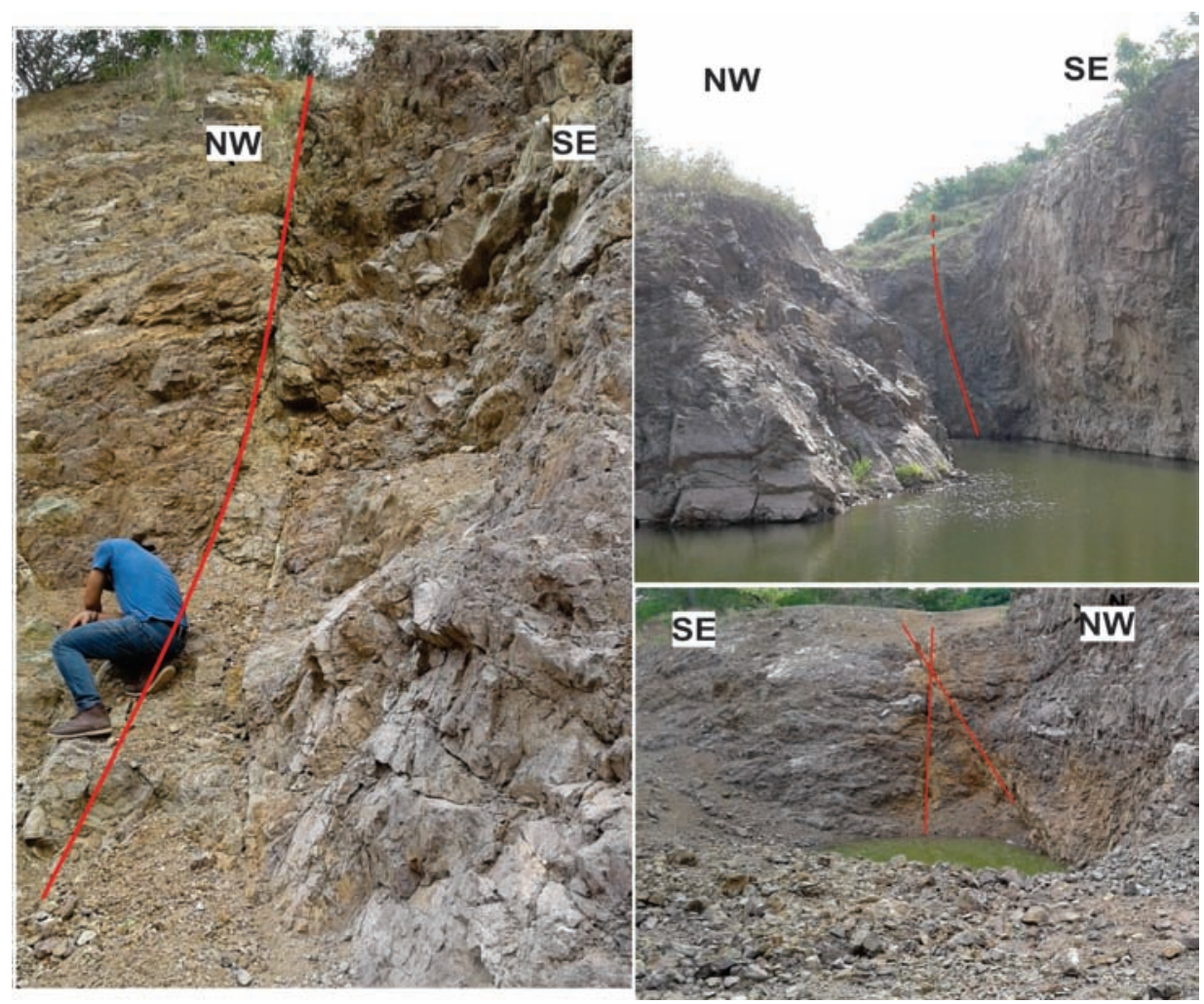

FIGURA 5. Aspecto en el terreno de los planos de fallas locales, localizado en Camperucho.

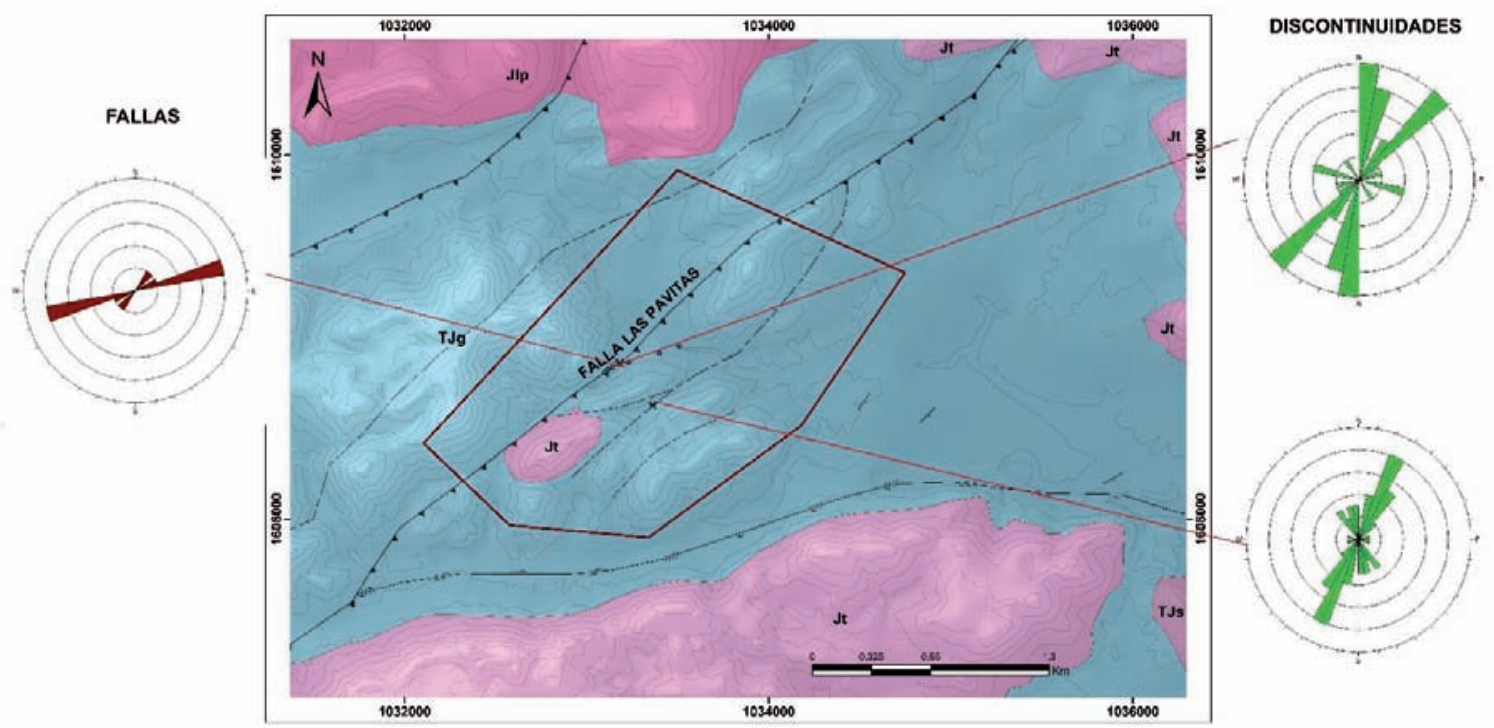

FIGURA 6. Mapa de la zona de estudio del corregimiento de Caracolí (recuadro rojo). En este se muestran las tendencias NESW de los principales planos de fallas $(\mathrm{n}=3)$ y diaclasas. Geología modificada de la plancha 33, escala 1:100 000 (Colmenares et al., 2007). 
En el corregimiento de Camperucho, se identificaron en función de la recolección de 122 datos de diaclasas, clasificadas en 5 familias diferentes: los rumbos de mayor frecuencia para las (diaclasas) N75E, N14W y N24W y los de menor frecuencia N26E y N74W; presentando buzamientos generales de $64^{\circ}, 51^{\circ}, 70^{\circ}$, $54^{\circ}$ y $16^{\circ}$ respectivamente. Las fallas se aprecian como zonas de debilidad, casi verticales con aperturas centimétricas de dirección preferencial NEE-SWW, con rumbo de $\mathrm{N} 84^{\circ} \mathrm{E}$ predominante y pequeñas fallas con rumbos de direcciones $\mathrm{N} 74^{\circ}-75^{\circ} \mathrm{W}$, buzando en ángulos que van desde lo $67^{\circ}-85^{\circ}$ (FIGURA 7).

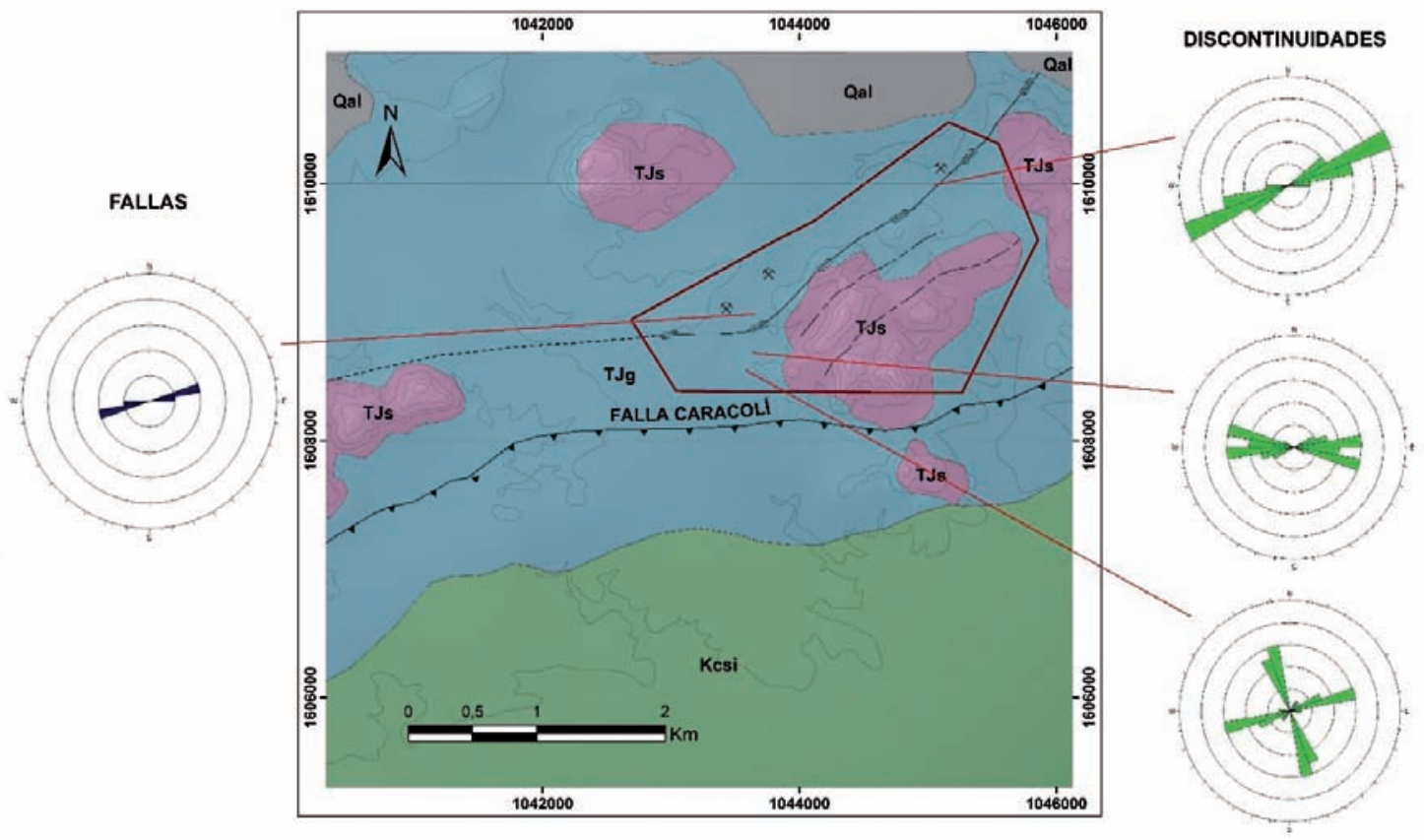

FIGURA 7. Mapa de la zona de estudio del corregimiento de Camperucho (recuadro rojo). Los datos muestran las tendencias NE-SW y W-E de los principales planos de fallas $(n=3)$ y diaclasas. Geología modificada de la plancha 33, escala 1:100 000 (Colmenares et al., 2007).

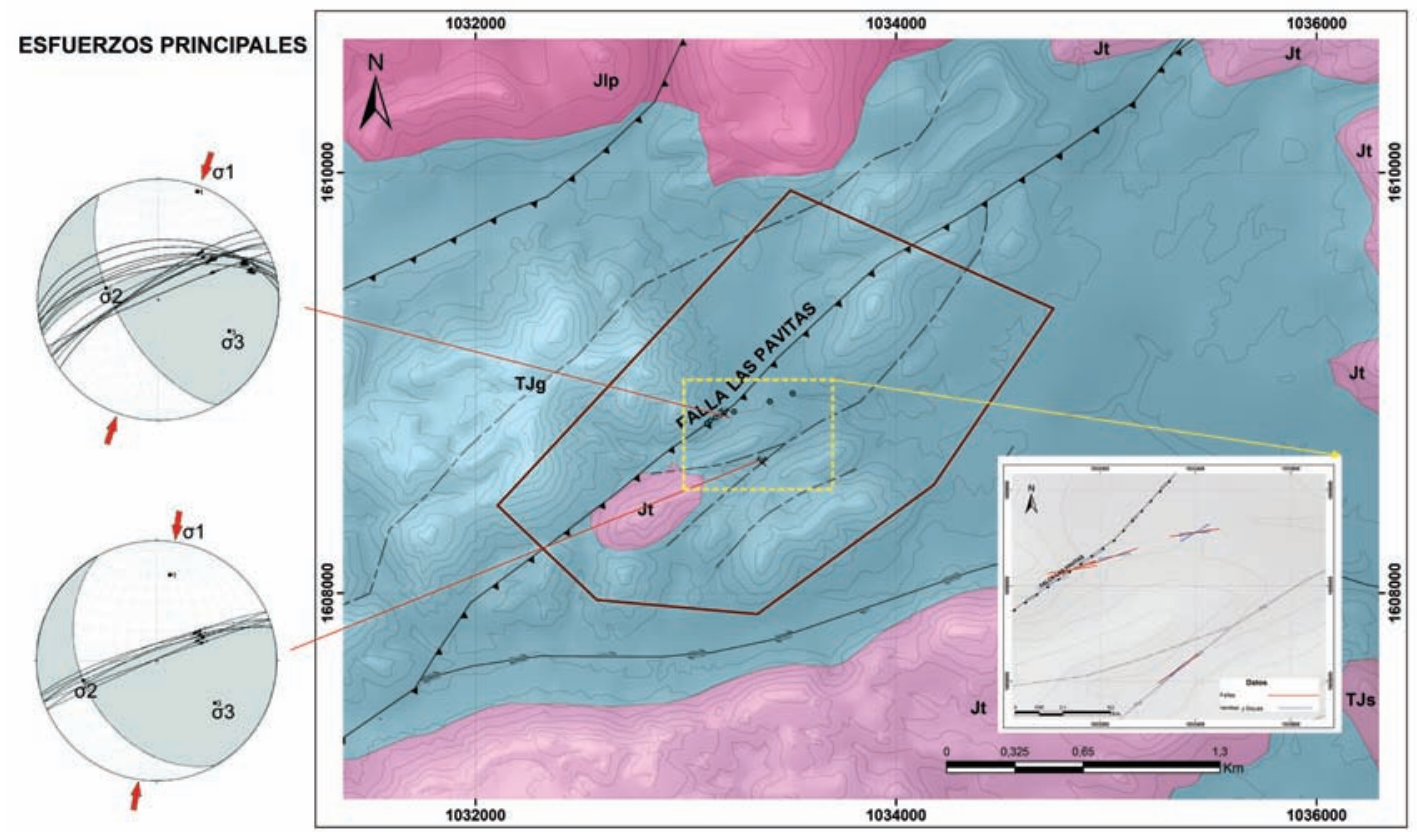

FIGURA 8. Mapa de la zona de estudio del corregimiento de Caracolí (recuadro rojo). Los datos muestran la dirección de los principales esfuerzos compresivos, con movimientos inversos y sinestrales. Geología modificada de la plancha 33, escala 1:100 000 (Colmenares et al., 2007). 
La zona evidencia esfuerzos compresivos y fallamientos con movimientos inversos y sinestrales según los datos cinemáticos medidos en campo. Los esfuerzos principales que generaron el tectonismo para el área de Caracolí son: $\sigma 1$ con un trend $17,6^{\circ}$ y un plunch de $13^{\circ}$, $\sigma 2$ con un trend de $271^{\circ}$ y un plunch de $51^{\circ}$ y $\sigma 3$ con un trend de $117,2^{\circ}$ y un plunch de $36^{\circ}$ (FIGURA 8). Para el área de Camperucho son: $\sigma 1$ con trend de $9,1^{\circ}$ y un plunch de $20,6^{\circ}, \sigma 2$ con un trend de $254,4^{\circ}$ y un plunch $48,1^{\circ}$ y $\sigma 3$ con un trend de $114,1^{\circ}$ y un plunch de $34,6^{\circ}$ (FIGURA 9).

Las mineralizaciones principales están distribuidas a lo largo de los planos de falla, como se observa en la FIGURA 10 y 11.

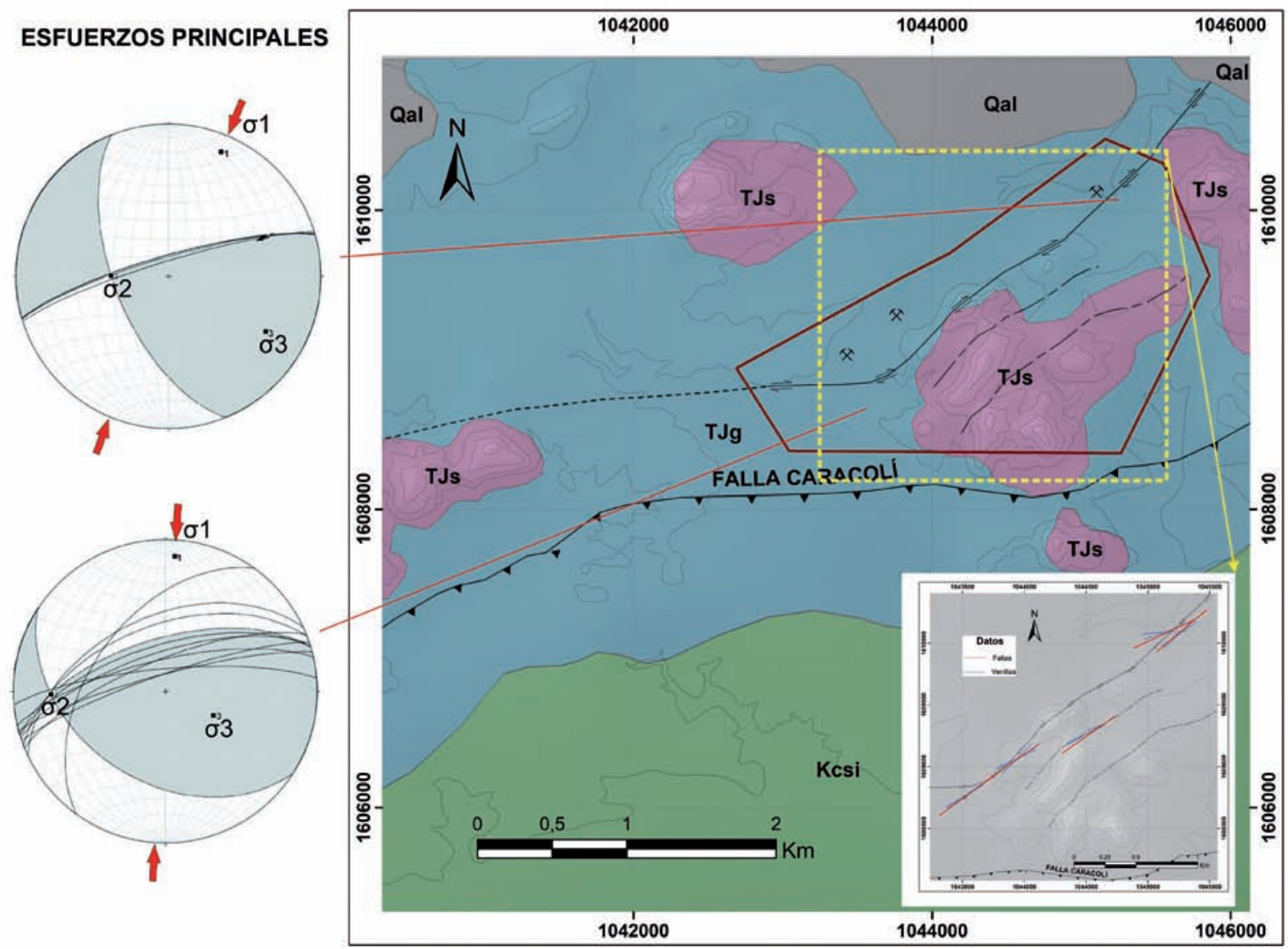

FIGURA 9. Mapa de la zona de estudio del corregimiento de Camperucho (recuadro rojo). Los datos muestran la dirección de los principales esfuerzos compresivos, con movimientos inversos y sinestrales. Geología modificada de la plancha 33 , escala 1:100 000 (Colmenares et al., 2007). 


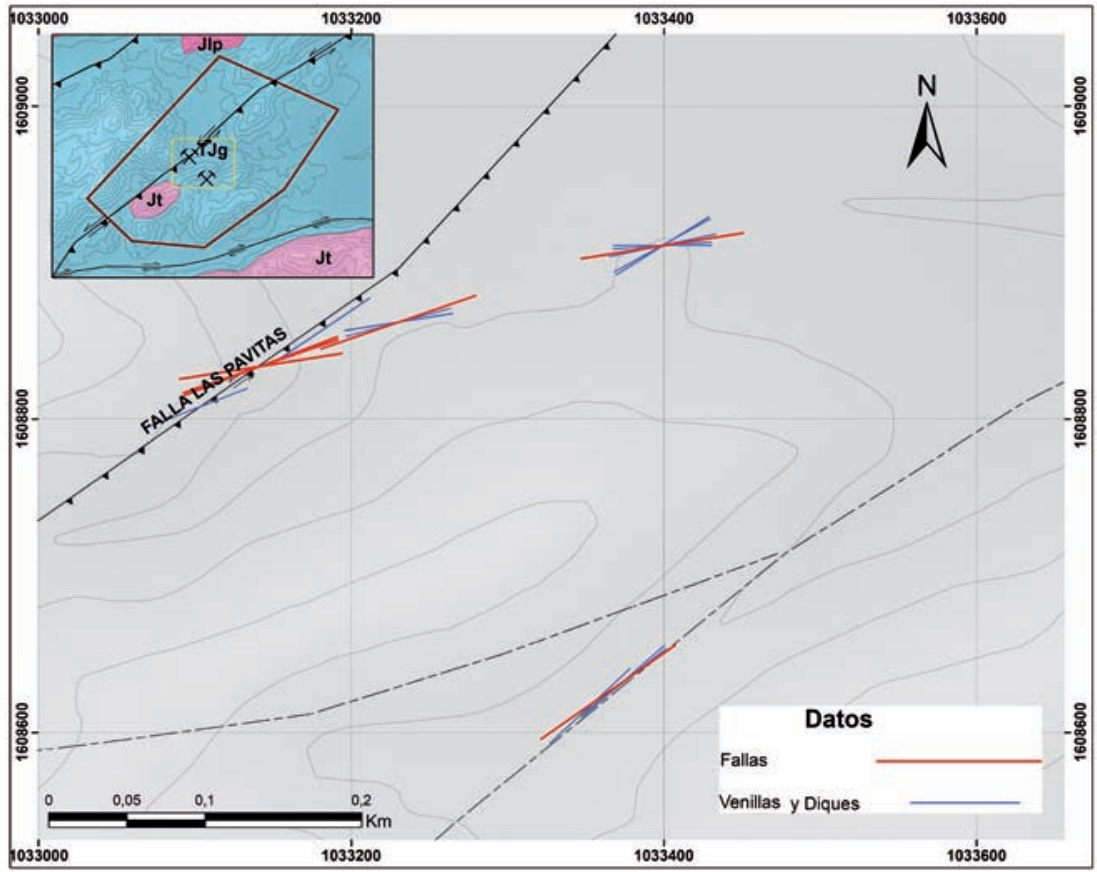

FIGURA 10. Mapa de distribución de fallas y mineralizaciones medidas en la zona de Caracolí, con orientaciones principales al NE-SW y NEE-SWW.

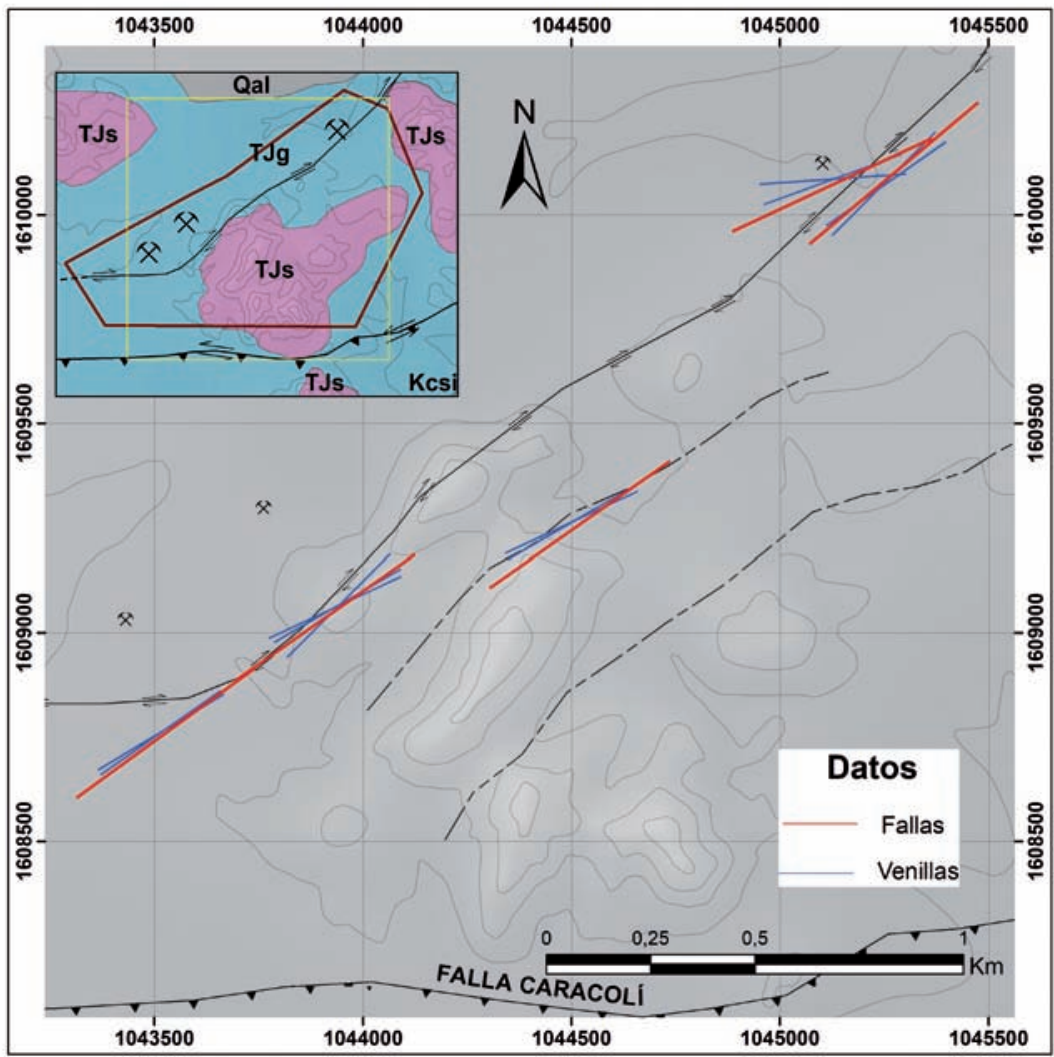

FIGURA 11. Mapa de distribución de fallas y mineralizaciones medidas en la zona de Camperucho, con orientaciones principales al NE-SW y NEE-SWW. 


\section{DISCUSIÓN}

La zona de estudio ha venido siendo explotada artesanalmente por más de 30 años extrayendo la barita, carbonatos y óxidos de cobre que han sido vendidos sin ningún tipo de beneficio mineral. Los pobladores de esta región han planeado sus explotaciones restringiéndose a seguir las vetas mineralizadas aflorantes. El levantamiento de datos estructurales y su respectivo análisis permitieron explicar ciertos aspectos de la distribución de las mineralizaciones, poniendo en evidencia la relación entre las mineralizaciones y las estructuras geológicas presentes. Los corregimientos de Caracolí y Camperucho se caracterizan por un estilo estructural compresivo (evidenciado en fallas inversas y plegamientos). Siendo estos posteriores a la litificación de la formación Guatapurí, y las unidades Riodacita de los Tábanos y Espilitas Triásicas de edades Triásico-Jurásico. Esos esfuerzos generaron un sistema de fallas y fracturas asociadas, receptivas al emplazamiento de venillas y vetas de barita (de naturaleza hidrotermal) en Caracolí y de óxidos y carbonatos de cobre (de naturaleza supergénica) en Camperucho. En la región, además, existen esfuerzos transcurrentes medidos en las direcciones de estrías de falla con direcciones de NE-SW a NEE-SWW para ambos corregimientos.

Los fallamientos en el área de Caracolí han funcionado como zonas de debilidad los cuales facilitaron el flujo y precipitación de mineralización de origen hidrotermal. Los planos de falla presentan curvaturas que permite a los movimientos transcurrentes tener zonas de cierre con fallamientos inversos y zonas de mayor apertura. Esto podría explicar la aparición de mineralizaciones de barita en formas de rosarios y bolsones, ya que estas mineralizaciones no son constantes sobre los planos de falla.

En el área de Camperucho las transcurrencias son muy poco sinuosas, lo cual no generó zonas con apertura apreciable, que restringieron las mineralizaciones de barita a solo unos pocos centímetros en el mejor de los casos. Sin embargo, fluidos meteóricos si usaron esos pequeños planos de debilidad para facilitar el desarrollo de procesos de enriquecimiento secundario. La aparición de la mineralización disminuye en la dirección NW a SE y está restringida a pequeñas manifestaciones alineadas a los planos de falla que no superan los $20 \mathrm{~cm}$ de espesor en las zonas más enriquecidas y $5 \mathrm{~cm}$ de espesor al $\mathrm{SE}$ en las zonas menos enriquecidas.

\section{CONCLUSIONES}

El tectonismo en la zona de Caracolí (localizado en las estribaciones sur de la Sierra Nevada de Santa Marta) ha sido reportado como compresivo bajo el actuar de Fallamientos inversos. Sin embargo, se ha identificado que los bloques desplazados tienen, además, movimientos transcurrentes sinestrolaterales evidenciados en las estrías de varios planos de falla medidos en campo.

Las mineralizaciones en la región se asocian a procesos hidrotermales confinados en planos de debilidad, en este caso fallamientos inversos y transcurrentes. Es evidente, además, que la zona de Camperucho al tener un tectonismo netamente transcurrente con planos de fallas rectos, no presentan mineralizaciones hidrotermales mayores, solo se evidencian mineralizaciones secundarias supergénicas.

\section{AGRADECIMIENTOS}

Agradecimientos a la Fundación Universitaria del Área Andina por su interés y apoyo en la investigación de las mineralizaciones de la Sierra Nevada de Santa Marta y Serranía del Perijá. Al Ingeniero Gustavo Daza Álvarez, quien nos permitió cada vez que era necesario el acceso al área de explotación de las mineralizaciones de Barita en Caracolí.

\section{REFERENCIAS}

Allmendinger, R.W., Cardozo, N., and Fisher, D. (2012). Structural geology algorithms: Vectors and tensors. New York: Cambridge University Press.

Bayona, G., Lamus-Ochoa, F., Cardona, A., Jaramillo, C., Montes, C., y Tchegliakova, N. (2007). Procesos orogénicos del Paleoceno para la cuenca de Ranchería (Guajira, Colombia) y áreas adyacentes definidos por análisis de procedencia. Geología Colombiana, 32, 21-46.

Colmenares, F., Mesa, A., Roncancio, J., Arciniegas, E., Pedraza, P., Cardona, A., Romero, A., Silva, C., Alvarado, S., Romero, O., y Vargas, A. (2007). Geología de las planchas 11, 12, 13, 14, 18, 19, 20, 21, 25, 26, 27, 33, 34 y 40. Proyecto: Evolución geohistórica de la Sierra Nevada de Santa Marta. INGEOMINAS. Bogotá. 
Corredor, F.H., y Mendoza, F.D. (2016). Caracterización mineralógica, geoquímica y génesis de las manifestaciones cupríferas de la zona Suroriental de la Sierra Nevada de Santa Marta, departamento del Cesar. Tesis, Universidad Industrial de Santander y Fundación Universitaria del Área Andina.

Etayo, F., Barrero, D., Lozano, H., Espinoza, A., González, H., Orrego, A., Ballesteros, I., Forero, H., Ramírez, C., Albers, J., Case, J., Singer, D., and Bowen, R. (1983). Mapa de Terrenos Geológicos de Colombia. Bogotá: Publicaciones Geológicas Especiales, INGEOMINAS, No 14.

IGAC. (2005a). Plano Topográfico 33-IV-A. Departamento del Cesar. Escala 1:25000. Instituto Geográfico Agustín Codazzi.

IGAC. (2005b). Plano Topográfico 33-IV-C. Departamento del Cesar. Escala 1:25000. Instituto Geográfico Agustín Codazzi.

Maestre, L., Toncel, H., y Rudas, A. (2014). Determinación de la calidad de los depósitos de Barita, en el corregimiento de Caracolí, municipio de Valledupar, Cesar. Revista Agunkuya, 14, 47-54.

Marrett, R.A., and Allmendinger, R.W. (1990). Kinematic analysis of fault-slip data. Journal of Structural Geology, 12(8), 973-986. doi: 10.1016/0191-8141(90)90093-E.

Montes, C., Guzman, G., Bayona, G., Cardona, A., Valencia, V., and Jaramillo, C. (2010). Clockwise rotation of the Santa Marta massif and simultaneous Paleogene to Neogene deformation of the PlatoSan Jorge and Cesar-Ranchería basins. Journal of South American Earth Sciences, 29(4), 832-848. doi: 10.1016/j.jsames.2009.07.010.
Ortega, C. (2011). Mineralización de Barita en volcánico mesozoicos en el municipio de Valledupar-Cesar, 6.2 Geología general.

Ortega, C., Rojas, E., y Manco, D. (2014). Mineralización de Cobre en el Sector de San Diego, Serranía del Perijá. Geología Colombiana, 37, 51-61.

Ortiz, H. (1991). Geología Minera del Oro de Veta. Texto Guía. COLCIENCIAS.

Reich, M., and Vasconcelos, P. (2015). Geological and economic significance of supergene metal deposits. Elements, 11(5), 305-310. doi: 10.2113/ gselements.11.5.305.

Tschanz, C., Jimeno, A., y Cruz, J. (1969). Mapa geológico de reconocimiento de la Sierra Nevada de Santa Marta y la Serranía del Perijá. INGEOMINAS.

Tschanz, C., Marvin R., Cruz J., Mehnert H., and Cebula, G. (1974). Geologic evolution of the Sierra Nevada de Santa Marta, Northeastern Colombia. GSA Bulletin, 85(2), 273-284. doi: 10.1130/0016-7606(1974)85<273:GEOTSN $>2.0$. $\mathrm{CO} ; 2$.

\begin{tabular}{c} 
Amat D. Zuluaga \\
ORCID: 0000-0002-0498-4698 \\
Wilfar Torrenegra \\
ORCID: 0000-0002-1455-4261 \\
Frank Lascarro \\
ORCID: 0000-0001-9433-2863 \\
\hline \hline
\end{tabular}

Trabajo recibido: noviembre 08 de 2017

Trabajo aceptado: agosto 22 de 2018 